\title{
Modeling and Real-timecontrol of MAV with Multi-rotors
}

\author{
Xiang-jian Chen ${ }^{1}$, Di $\mathrm{Li}^{2}$, Zhi-jun $\mathrm{Xu}^{3}$ and Yue $\mathrm{Bai}^{3}$ \\ ${ }^{1}$ Jiangsu University of science and technology, School of computer science and \\ Engineering, China \\ ${ }^{2}$ China Shipbuilding Industry Corporation 723, China \\ ${ }^{3}$ Chang Chun Institute of optics fine mechanics and physics, Chinese academy of \\ sciences, China \\ cxj831209@163.com,lidi19821111@163.com
}

\begin{abstract}
We introduce one configuration of a multi rotor Micro Aerial Vehicle (MAV) composed of six rotors. Accurate modeling and robust flight control of the SixRotor MAV are discussed in this work. Rigorous dynamic model of the SixRotor MAV is obtained both in reference and body frame coordinate systems. The main characteristic of this configuration is the gyroscopic effects and aerodynamic torques tend to be cancelled in the trimmed flight due to the unique mechanical structure, and the attitude and translation dynamics are decoupled which is benefit for designing the control scheme. A disturbance compensator based controller using the derived dynamic models is proposed here for robust hovering control. The disturbance compensator based controller designed by regarding the inaccurate part of the model and sensor noises as disturbances. The validity of the proposed control method has been verified through real-time experiments. The experimental results show that the performance of disturbance compensator based controller performs very well under sensor noise and external disturbances, and have more superiority than traditional PID controller.
\end{abstract}

Keywords: Micro Aerial Vehicle with six rotors; disturbance compensator

\section{Introduction}

The development of micro aerial vehicles (MAVs) has generated great interest in the automatic control area in the last few decades. These kinds of vehicles have been used in tasks such as search and rescue, building exploration, security, military inspection [1]. The MAV are most useful, mainly, when these desired tasks are executed in dangerous and inaccessible environments. Future MAVs will evolve form being robots operated at a distance to independent robots, able to self-actualize to perform a given task. This ability, autonomy, has many levels emerging by which it is defined, but ultimate autonomy will require capabilities analogous to those of the human brain by future MAV mission management computers.

MAVs are categorized by size and mission performance. Of course, the whole purpose of the MAV is to carry a payload. For the moment, the primary mission assigned to MAVs is aerial observation and surveillance. In general, for MAVs to carry out the missions under consideration, all aspects of the system will have to be improved: the vehicle itself, payloads and especially sensors, transmission systems, onboard intelligence, sharing authority with human operator and data processing and sensor fusion. 
The major limitation of helicopters is the need for extensive, and costly, maintenance for reliable operation. MAV rotorcraft is no exception. Simplifying the mechanical structure of such craft clearly produces logistical benefits. Quadrotor MAV [2-6] has been popular for its simplified mechanism. However, it must be taken into account that this vehicle is under actuated mechanical systems which complicate the control design stage even more.

We introduce, in this paper, a configuration of a multi rotor helicopter composed of six rotors. SixRotor MAV [7] is an alternative form of rotorcraft which does not have the complicated swash plates and linkages found in conventional designs, and instead use varying rotor speeds to maneuver, the other advantage of the SixRotor MAV configuration is its load capacity. Due to the great reduction of mechanical complexity and wear, it is expected that well-designed SixRotor will prove inherently more robust and reliable. The main characteristic of this configuration is that the attitude and translation dynamics are almost totally decoupled. We extend our results and present the nonlinear model taking into account aerodynamics effects. To validate the model we propose a control strategy based on disturbance compensator and we apply it in realtime experiences.

\section{Dynamic of MAV with sixrotor}

All printed material, including text, illustrations, and charts, must be kept within the parameters of the 8 15/16-inch (53.75 picas) column length and 5 15/16-inch (36 picas) column width. Please do not write or print outside of the column parameters. Margins are $15 / 16$ of an inch on the sides ( 8 picas), 7/8 of an inch on the top (5.5 picas), and 1 $3 / 16$ of an inch on the bottom (7 picas).

Mathematical dynamic models of flight behavior are essential for good control design and analysis. In this subsection we will describe the dynamical models if the SixRotor MAV. See Figure 1 shows coordinate systems of SixRotor MAV. The motion is induced by the combination of six rotors. The SixRotor MAV has some advantages over conventional helicopter in terms of simplicity of dynamics and control design. Given that each near rotors rotate counter-clockwise, and the rotors form $\varpi$ degree with the earth coordinate system, then the gyroscopic effects and aerodynamic torques tend to be cancelled in the trimmed flight. This MAV does not have a swashplate. In fact, it does not need any blade pitch control. The collective input is the sum of the thrust of individual rotors which results in simple dynamics. Meanwhile, SixRotor MAV enables independent control of three degrees of freedom in translation and three degrees of freedom in orientation with the unique mechanical design. The state rectors for the SixRotor MAV are described as following:

$$
\begin{aligned}
\eta & =[x, y, z, \phi, \theta, \psi]^{T} \\
v & =\left[v_{x}, v_{y}, v_{z}, w_{x}, w_{y}, w_{z}\right]^{T}
\end{aligned}
$$

Where the position and orientation of SixRotor MAV, $\eta$, are described relative to the inertial reference frame, while the linear and angular velocities of SixRotor MAV, v, are expressed in the body-fixed frame. $\mathrm{x}, y, z$ mean the linear positions with respect to inertial reference frame. $\phi, \theta, \psi$ respect the roll, yaw, and pitch angels of SixRotor MAV in the inertial reference frame. 


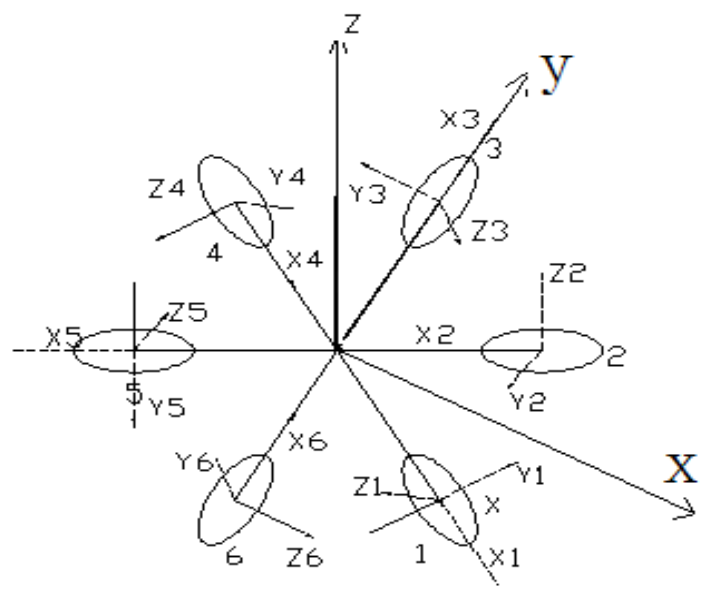

Figure 1. Coordinate systems of SixRotor MAV

The inertial and body-fixed velocity relationship can be represented with SixRotor MAV Jacobian matrix as following:

$$
\begin{aligned}
& \dot{\eta}=\mathrm{J}(\eta) v \\
& \mathrm{~J}(\eta)=\left[\begin{array}{ccclcc}
c \psi c \theta & -s \psi c \phi+c \psi s \theta s \phi & s \psi s \phi+c \psi c \phi s \theta & 0 & 0 & 0 \\
s \psi c \theta & c \psi c \phi+s \psi s \theta s \phi & -c \psi s \phi+s \psi s \phi c \theta & 0 & 0 & 0 \\
-s \theta & c \theta s \phi & c \theta c \phi & 0 & 0 & 0 \\
0 & 0 & 0 & 1 & s \phi t \theta & c \phi t \theta \\
0 & 0 & 0 & 0 & c \phi & -s \phi \\
0 & 0 & 0 & 0 & s \phi / c \theta & c \phi / c \theta
\end{array}\right]^{T}
\end{aligned}
$$

In above equations, $\mathrm{c}(\bullet), s(\bullet)$ denote $\cos (\bullet), \sin (\bullet)$ respectively. For the moving base system which is not fixed in an inertial frame, it is not convenient to derive the dynamic formulation using the Lagrangian in terms of the velocities expressed in a body-fixed frame. We used quasi-Lagrange method which can give the equations of motion in terms of the body-fixed velocities.

\subsection{Quasi-Lagrange Equations of Motion}

The Lagrangian method [6] in general form is defined as:

$\mathrm{L}=T-V$

$T=\frac{1}{2} \mathrm{v}^{T} m \mathrm{v} ;$

$V=-m g z$

Normally, we design the SixRotor MAV body as axisymmetric so the inertia is defined as Eq.5, and especially $\mathrm{I}_{x x}=I_{y y}$. 


$$
\mathrm{I}=\left[\begin{array}{ccc}
I_{x x} & 0 & 0 \\
0 & I_{y y} & 0 \\
0 & 0 & I_{z z}
\end{array}\right]
$$

And with the Eq.6:

$$
M \dot{v}+C v+g=\tau
$$

Finally, the 6 independent equations of motions are obtained as following:

$$
\left\{\begin{array}{l}
\mathrm{m}\left[\dot{v}_{x}-v_{y} w_{z}+v_{z} w_{y}-g s \theta\right]=u_{1} \\
\mathrm{~m}\left[\dot{v}_{y}-v_{z} w_{x}+v_{x} w_{z}-g c \theta s \phi\right]=u_{2} \\
\mathrm{~m}\left[\dot{v}_{z}-v_{y} w_{y}+v_{y} w_{x}-g c \theta c \phi\right]=u_{3} \\
I_{x x} \dot{w}_{x}+\left(I_{z z}-I_{y y}\right) w_{y} w_{z}=u_{4} \\
I_{y y} \dot{w}_{y}+\left(I_{x x}-I_{z z}\right) w_{z} w_{x}=u_{5} \\
I_{z z} \dot{w}_{z}=u_{6}
\end{array}\right.
$$

Where,

$$
\tau=\left[\begin{array}{l}
\mathrm{u}_{1} \\
\mathrm{u}_{2} \\
\mathrm{u}_{3} \\
\mathrm{u}_{4} \\
\mathrm{u}_{5} \\
\mathrm{u}_{6}
\end{array}\right]=\left[\begin{array}{c}
1 / 2 k_{1} \cos \omega\left(-F_{1}-F_{2}+2 F_{3}-F_{4}-F_{5}+2 F_{6}\right) \\
\sqrt{3} / 2 k_{1} \cos \omega\left(-F_{1}+F_{2}-F_{4}+F_{5}\right) \\
k_{1} \sin \omega\left(F_{1}+F_{2}+F_{3}+F_{4}+F_{5}+F_{6}\right) \\
1 / 2\left(k_{1} l \sin \pi-k_{2} \cos \pi\right)\left(-F_{1}+F_{2}+2 F_{3}+F_{4}-F_{5}-2 F_{6}\right) \\
\sqrt{3} / 2\left(-k_{1} l \sin \omega+k_{2} \cos \pi\right)\left(F_{1}+F_{2}-F_{4}-F_{5}\right) \\
\left(-k_{1} l \cos \omega-k_{2} \sin \omega\right)\left(F_{1}-F_{2}+F_{3}-F_{4}+F_{5}-F_{6}\right)
\end{array}\right]
$$

\subsection{Earth-fixed vector representation}

Until now, we derive the dynamic equation of motion relative to the body-fixed coordinate frame. However, for the control purpose, it is more convenient to use the dynamic equations derived in earth-fixed coordinate frame like below:

$$
\mathrm{M}_{\eta}(\eta) \ddot{\eta}+C_{\eta}(v, \eta)(\eta)+g_{\eta}(\eta)=\tau_{\eta}(\eta)
$$

To express the dynamic equations in earth-fixed coordinate frame like Eq.9, we need following relationship:

$$
\begin{aligned}
& \dot{\eta}=J(\eta) v \Leftrightarrow v=J^{-1}(\eta) \dot{\eta} \\
& \ddot{\eta}=J(\eta) \dot{v}+\dot{\eta}(\eta) v \Rightarrow \dot{v}=J^{-1}(\eta)[\ddot{\eta}-\dot{J}(\eta) v]
\end{aligned}
$$


Then, the system matrices are defined as following:

$$
\begin{aligned}
& \mathrm{M}_{\eta}(\eta)=J^{-T}(\eta) M J^{-1}(\eta) ; \\
& C_{\eta}(v, \eta)=1 / 2 \dot{M}_{\eta}(\eta) ; \\
& g_{\eta}(\eta)=J^{-T}(\eta) g(\eta) ; \\
& \tau_{\eta}(\eta)=J^{-T}(\eta) \tau ;
\end{aligned}
$$

Finally, we can derive the equations of motion in earth-fixed coordinate frame as followings:

Where

$$
\begin{aligned}
& \left\{\begin{array}{l}
m \ddot{x}=c \phi c \theta u_{1} \\
m \ddot{y}=(c \psi c \phi+s \psi s \theta s \phi) u_{2} \\
m \ddot{z}=c \theta c \phi u_{3} \\
M_{2} \ddot{\eta}+\frac{1}{2} \dot{M}_{2} \dot{\eta}=\left[\begin{array}{l}
u_{3} \\
u_{4} c \phi-u_{5} s \phi \\
-u_{4} s \theta+u_{5} c \theta s \phi+u_{6} c \theta c \phi
\end{array}\right]
\end{array}\right. \\
& M_{2}=\left[\begin{array}{ccc}
I_{x x} & 0 & -I_{x x} s \theta \\
0 & I_{y y} c^{2} \theta+I_{z z} s^{2} \phi & \left(I_{y y}-I_{z z}\right) c \phi c \theta s \phi \\
-I_{x x} s \theta & \left(I_{y y}-I_{z z}\right) c \phi c \theta s \phi & I_{x x} s^{2} \theta+I_{y y} c^{2} \theta s^{2} \phi+I_{z z} c^{2} \theta c^{2} \phi
\end{array}\right]
\end{aligned}
$$

\subsection{Equations of Motion of SixRotor MAV}

According to the above analysis, the following equations are derived:

$$
\left\{\begin{array}{l}
m \ddot{x}=c \phi c \theta u_{1} \\
m \ddot{y}=(c \psi c \phi+s \psi s \theta s \phi) u_{2} \\
m \ddot{z}=c \theta c \phi u_{3} \\
I_{x x} \dot{w}_{x}+\left(I_{z z}-I_{y y}\right) w_{y} w_{z}=u_{4} \\
I_{y y} \dot{w}_{y}+\left(I_{x x}-I_{z z}\right) w_{z} w_{x}=u_{5} \\
I_{z z} \dot{w}_{z}=u_{6}
\end{array}\right.
$$

As show in Eq.14, for the linear motions and angular motions, all the states are subordinated to the control parameters $u_{1} \sim u_{6}$. In this paper, we analyze the vertical up-down and hovering control with $\theta \approx 0 ; \phi \approx 0 ; \psi \approx 0$ which can make the dynamics much simpler form like Eq.15, and it is easy to design the controller: 


$$
\left\{\begin{array}{l}
m \ddot{x}=u_{1} \\
m \ddot{y}=u_{2} \\
m \ddot{z}=u_{3} \\
I_{x x} \dot{w}_{x}=u_{4}-\left(I_{z z}-I_{y y}\right) \dot{\theta} \dot{\phi} \\
I_{y y} \dot{w}_{y}=u_{5}-\left(I_{x x}-I_{z z}\right) \dot{\psi} \dot{\phi} \\
I_{z z} \dot{w}_{z}=u_{6}
\end{array}\right.
$$

\section{Controller Design}

Now, let us consider following composite dynamic equations of motion:

$$
\left[\begin{array}{l}
m \ddot{x} \\
m \ddot{y} \\
m(\ddot{z}+g) \\
I_{x x} \ddot{\phi} \\
I_{y y} \ddot{\theta} \\
I_{z z} \ddot{\psi}
\end{array}\right]+\Delta=\left[\begin{array}{l}
u_{1} \\
u_{2} \\
u_{3} \\
u_{4} \\
u_{5} \\
u_{6}
\end{array}\right]
$$

Where, the disturbance $\Delta$ is defined as

$$
\Delta=\left[\begin{array}{l}
\delta_{1} \\
\delta_{2} \\
\delta_{3} \\
\delta_{4}+\left(I_{z z}-I_{y y}\right) \dot{\theta} \dot{\psi} \\
\delta_{5}+\left(I_{x x}-I_{z z}\right) \dot{\psi} \dot{\phi} \\
\delta_{6}
\end{array}\right]
$$

And $\delta_{\mathrm{i}}$ mainly comes from dynamic inconsistency, the control algorithms for the position and attitude of the SixRotor MAV are designed based on PID controllers as shown in Figure 2. The control inputs $u_{i}(i=1,2,3)$ for controlling the position $(x, y, z)$ of the SixRotor with respect to the reference input $x_{d}, y_{d}, z_{d}$ are designed as:

$$
\begin{gathered}
u_{i}=K_{p i}\left[(x, y, z)_{d}-(x, y, z)\right]+K_{d i} \frac{d\left[(x, y, z)_{d}-(x, y, z)\right]}{d t}+ \\
K_{\mathrm{ii}} \int_{0}^{t}\left[(x, y, z)_{d}-(x, y, z)\right] d \tau
\end{gathered}
$$

The control inputs $u_{j}(j=4,5,6)$ for controlling the attitude $(\phi, \theta, \psi)$ of the SixRotor with respect to the reference input $\phi_{d}, \theta_{d}, \psi_{d}$ are designed as: 


$$
\begin{aligned}
u_{j}= & K_{p j}\left[(\phi, \theta, \psi)_{d}-(\phi, \theta, \psi)\right]+K_{d \mathrm{j}}(\dot{\phi}, \dot{\theta}, \dot{\psi}) \\
& +K_{\mathrm{ij}} \int_{0}^{t}\left[(\phi, \theta, \psi)_{d}-(\phi, \theta, \psi)\right] d \tau
\end{aligned}
$$

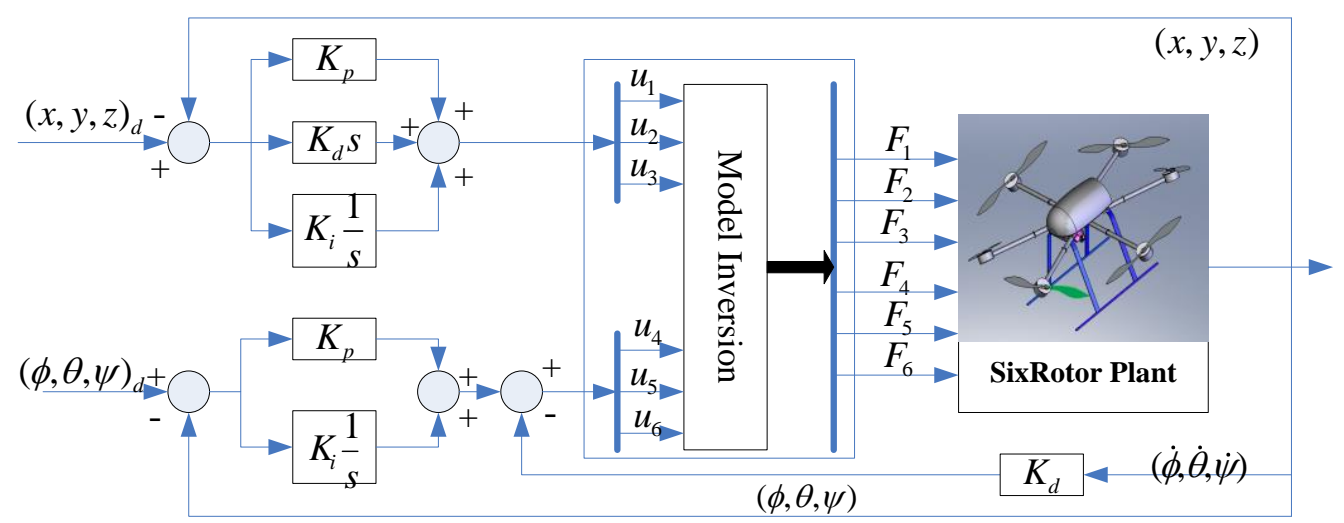

Figure 2. PID controller for controlling position and attitude of SixRotor MAV

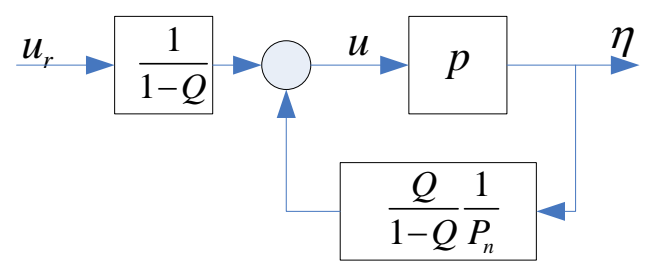

Figure 3. Disturbance compensator

The above control algorithms Eqs.18 and 19 control the position and angular of the MAV using sensor signals. The flight performance is not good in real flight because of the sensor noises and disturbances. The disturbance compensator based controller as shown in Figure 3, controlling the response of the plant $\mathrm{P}(\mathrm{s})$ to follow that mode plant $P_{n}(s)$ even though disturbances, sensor noises, and modeling uncertainties are applied to the plant. The Disturbance compensator can be expressed as:

$$
u(s)=\frac{1}{1-Q} u_{r}(s)-\frac{Q}{1-Q} \frac{1}{P_{n}} \eta(s)
$$

The nominal models are given as $P_{n}(s)=\frac{1}{m s^{2}}$ for the linear motion, and $P_{n}(s)=\frac{1}{\left(I_{x x}, I_{y y}, I_{z z}\right) s^{2}}$ for the angular motion. And, for the Q-filter, we use well-kown $Q_{31}$ filter as following:

$$
Q_{31}(s)=\frac{1+3 \kappa s}{(1+\kappa s)^{3}}
$$


Then, the control loop will be converted as:

$$
\left[\begin{array}{l}
m \ddot{x} \\
m \ddot{y} \\
m(\ddot{z}+g) \\
I_{x x} \ddot{\phi} \\
I_{y y} \ddot{\theta} \\
I_{z z} \ddot{\psi}
\end{array}\right]=\left[\begin{array}{l}
u_{r 1} \\
u_{r 2} \\
u_{r 3} \\
u_{r 4} \\
u_{r 5} \\
u_{r 6}
\end{array}\right]
$$

Using the Eq.22, we can easily design the linear controller for each axis independently.

\section{References Aerial Control Platform and Real-time Implantation}

In this section, we describe the SixRotor MAV aerial control platform which is composed of on-board computer, power interface, IMU and present the real-time implementation of the developed control strategy.

\subsection{Aerial control platform}

Figure 4 shows the prototype of the SixRotor MAV.The SixRotor MAV has 6 blades driven by 6 BLDC motor mounted at each end of the body frame. The Encoders were used for measuring the speed of each motor. The GPS data are updated at $5 \mathrm{~Hz}$ and the static pressure sensor measures are provided at a rate of $50 \mathrm{~Hz}$, the low-cost IMU which outputs raw data from 3 accelerometers, 3 gyro meters and 3 magnetometers at the need of $50 \mathrm{~Hz}$ to the flight control computer which receives all these sensor data through an RS-232 serial port. The onboard flight control computer is TMS320F2812 (DSP) which runs at $29.4 \mathrm{M} \mathrm{Hz}$, with $512 \mathrm{k}$ flash memory, including eight serial ports, eight channels with programmable gains, 24-bit analog input, six programmable Pulse Width Modulation (PWM) outputs, and supports floating point calculations. Figure 4 shows the prototype of the SixRotor MAV.

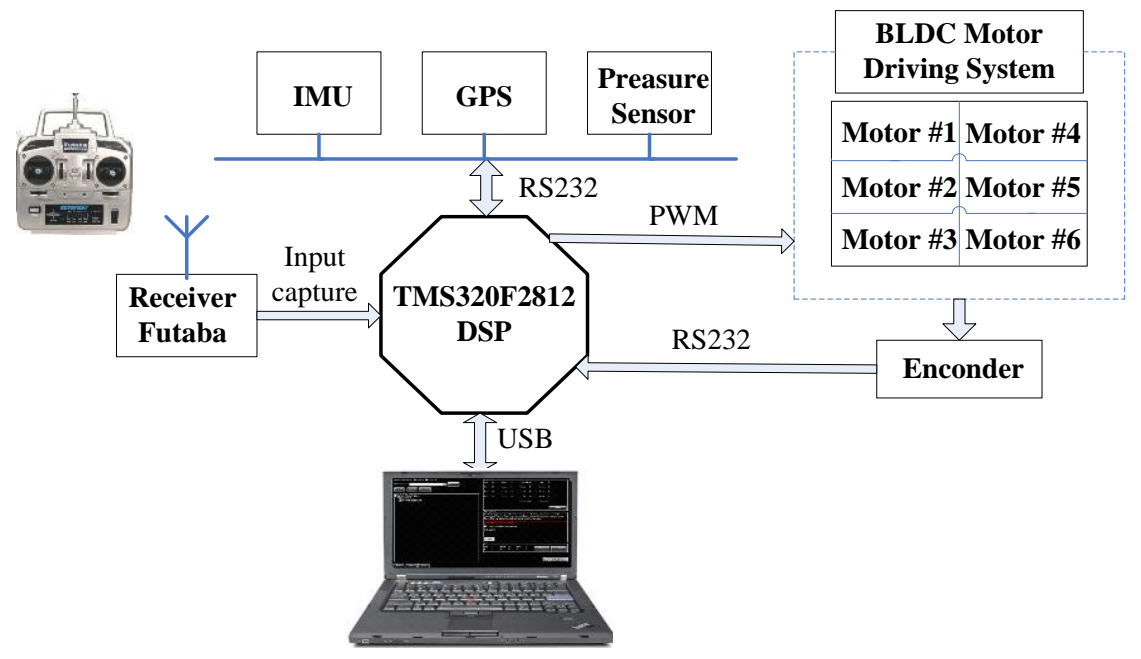

Figure 4. Schematic view of aerial control platform using TMS320F2812 DSP 
For avoiding signal interference between sensor signals and the motors PWM, two independent power supplies was supplied. One battery is used to feed the six electric motors which are controlled using PWM, the other battery is used to feed microcontroller and the sensors, and by adequate grounding, the interference is reduced largely.

\subsection{Real-time software}

The implemented software contains both real-time control software and ground station software. The real-time control software is implemented on the on-board computer, which updates the attitude variables at $50 \mathrm{~Hz}$ by fusing IMU raw data using EKF, and fuses the GPS and INS data in $10 \mathrm{~Hz}$ to provide the position and velocity estimates. The estimated state vector is then used by flight controller proposed here for MAV stabilization and trajectory tracking tasks, the control process runs at $50 \mathrm{~Hz}$ and provides six control commands (x thrust, $\mathrm{y}$ thrust, $\mathrm{z}$ thrust, yaw torque, pitch torque, roll troque) for the MAV.

The ground station requires one free USB port and a computer running Windows XP. View real-time streaming state while monitoring the SixRotor MAV gauges, and capture the incoming state vector to a digital file. The Figure 5 shows the configuration screen of SixRotor MAV ground station software.

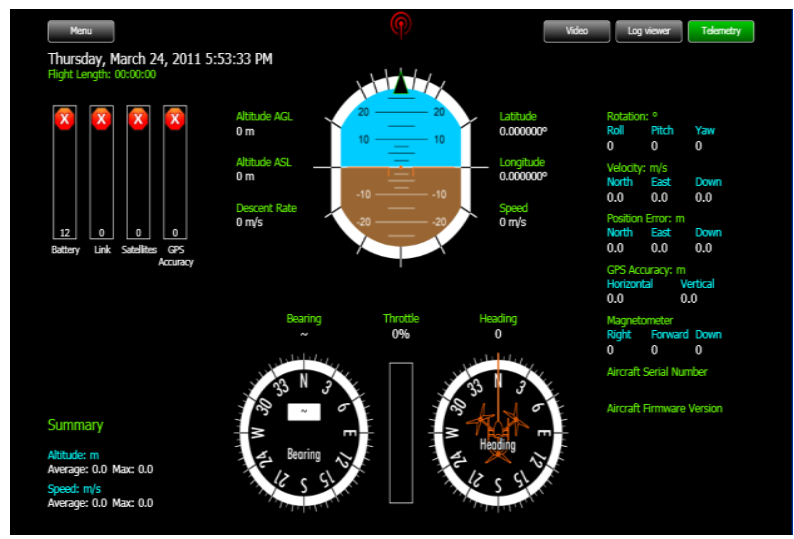

Figure 5. Configuration screen of SixRotor MAV ground station software

\section{Experimental Results}

\subsection{Real-time experiment results}

Real-time experiment results are presented in this section to validate the performance o the SixRotor MAV during hover flight. The control gains of the equations were adjusted in practice to obtain a fast aircraft response but avoiding mechanical oscillations as much as possible. The parameters were also chosen in such a way that the aircraft attitude remains very close to a desired point. 
International Journal of Control and Automation Vol.6, No.5 (2013)
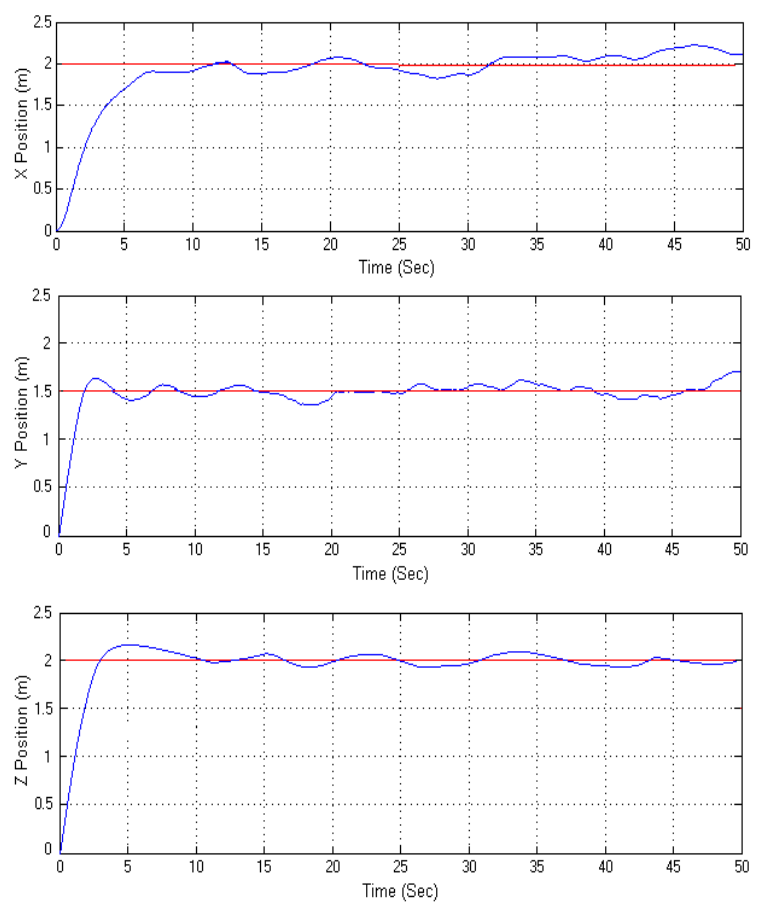

Figure 6. $\xi$-Position of the SixRotor MAV
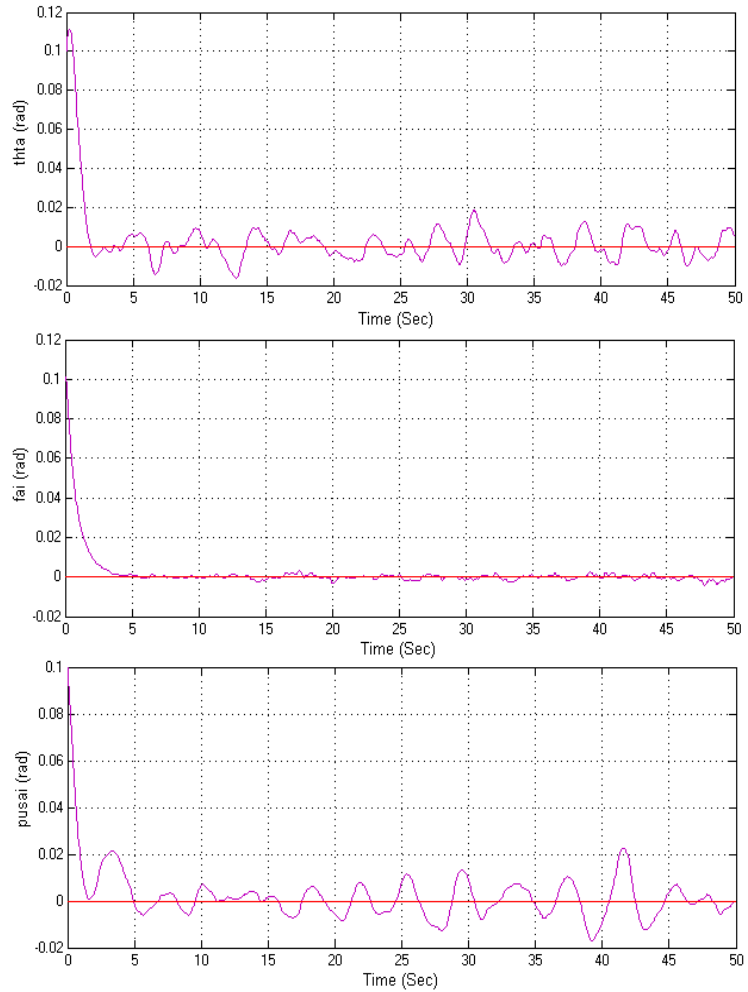

Figure 7. $\eta$-Angles of the SixRotor MAV 
In the experiment, the SixRotor MAV is stabilized at hover applying the proposed control strategy; we have obtained an acceptable which is shown in Figures 6 and 7. The control objective is to make the MAV hover while $\xi=(2,1.5,2) \mathrm{m}$ and $\eta=(0,0,0)^{\circ}$, the initial angle vector is set to be $\eta=(0.1,0.1,0.1)^{\circ}$ for validating the robustness of the proposed control strategy. Figure 8 shows the speed of the six rotors applied to the MAV.

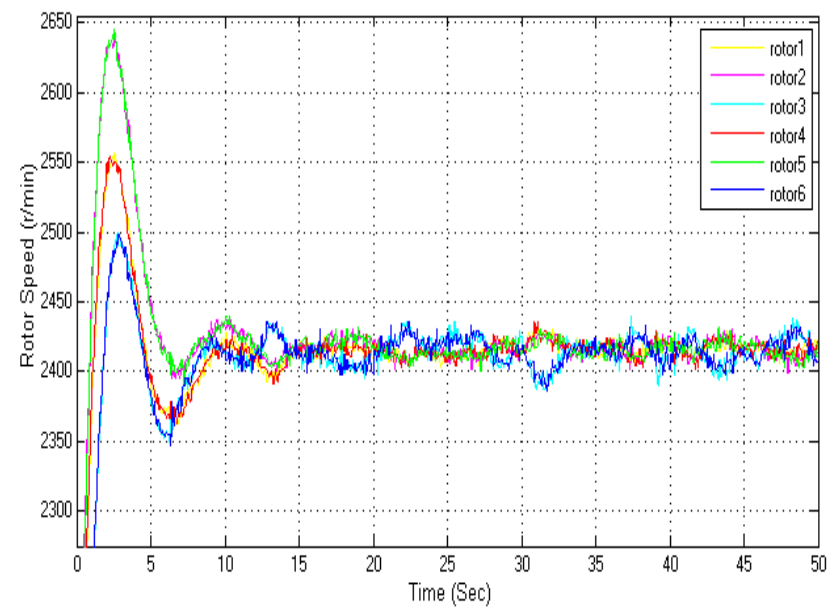

Figure 8. The speed of the six rotors

\section{Real-time Experiment Competitions}

Experimental results using disturbance compensator based controller are compared with those from the PID controller for varied flight conditions and under the influence of external disturbance. To test the controller in experiments for robustness, the sensor noise and effects of gusts are included.

The desired tracking trajectory follows:

$$
\left\{\begin{array}{l}
\psi=25^{\circ} \\
\theta=10^{\circ} \quad 0 \leq t \leq 400 \mathrm{~ms} \\
\phi=5^{\circ}
\end{array}\right.
$$

Table 1. Performance competition of two controllers

\begin{tabular}{cccc}
\hline & $\begin{array}{c}\text { Regulation } \\
\text { time(ms) }\end{array}$ & $\begin{array}{c}\text { Overshoot } \\
(\%)\end{array}$ & $\begin{array}{c}\text { steady-state } \\
\text { error }\left(^{\circ}\right)\end{array}$ \\
\hline Yaw1 & $180 / 245$ & $14 / 24.5$ & $0.05 / 0.30$ \\
Yaw2 & $145 / 150$ & $4.5 / 14$ & $0.08 / 0.10$ \\
Pitch1 & $205 / 240$ & $15.5 / 18.5$ & $0.03 / 0.25$ \\
Pitch2 & $120 / 145$ & $1.5 / 4.0$ & $0.045 / 0.100$ \\
Roll1 & $190 / 250$ & $8.8 / 28$ & $0.01 / 0.40$ \\
Roll2 & $100 / 120$ & $1 / 3$ & $0.01 / 0.05$ \\
\hline
\end{tabular}

The control performances by using disturbance compensator based controller and PID controller have been shown in Table1. From Table 1, the data before symbol "/"represents the flight performance without disturbances and other uncertainties; the data after symbol " $/$ " represents the flight performance under the disturbances and considering the uncertainties. 
"1" represents the PID controller; "2" represents the disturbance compensator based controller. It is obvious that the disturbance compensator based controller performs faster than PID controller and has less overshoot.

\section{Conclusion}

In this paper, we have presented the stabilization of a MAV having six rotors. One of the main characteristics of the configurations is that the translational and rotational dynamics are almost decoupled. Therefore we are able to apply the simple control strategy based disturbance observer to compensate the remaining dynamical coupling and to achieve the aircraft stabilization and location. The control algorithm is affordable for implementation in real-time applications. The real-time experiments have shown and acceptable performance of the helicopter applying the proposed control strategy.

\section{References}

[1] D. Lyon, "A military perspective on small unmanned aerial vehicles", IEEE Instrumentation and Measurement Magazine, vol. 7, no. 4, (2004), pp. 27-31.

[2] G. M. Hoffmann, H. Huang, S. L. Waslander and C. J. Tomlin, "Precision flight control for a multi-vehicle quadrotor helicopter testbed", Control Engineering Practice, vol. 19, no. 9, (2011), pp. 1023-1036.

[3] O. Alexis, G. Nikolakopoulos and A. Tzes, "Switching model predictive attitude control for a quadrotor helicopter subject to atmospheric disturbances", Control Engineering Practice, vol. 19, no. 10, (2011), pp. 1195-1207.

[4] C. Nicol, C. J. B. Macnab and A. Ramirez-Serrano, "Robust adaptive control of a quadrotor helicopter", Mechatronics, vol. 21, no. 6, (2011), pp. 927-938.

[5] J. F. Guerrero-Castellanos, N. Marchand, A. Hably, S. Lesecq and J. Delamare, "Bounded attitude control of rigid bodies: Real-time experimentation to quadrotor mini-helicopter", Control Engineering Practice, vol. 19, no. 8, (2011), pp. 790-797.

[6] Y. Yali, S. Feng and W. Yuanxi, "Controller Design of Quadrotor Aerial Robot", Physics Procedia, vol. 33, (2012), pp. 1254-1260.

[7] G. Xun, B. Yue, Z. Chang-jun, G. Qing-jia, P. Cheng and T. Yan-tao, "Hex-Rotor aircraft and its autonomous flight control system", Optics and Precision Engineering, vol. 20, no. 11, (2012), pp. 2451-24580. 